\title{
The Remarkable Evolution of the Post-AGB Star FG Sge
}

\author{
Johanna Jurcsik ${ }^{\mathrm{a}, 1}$ Benjamín Montesinos ${ }^{\mathrm{b}, 2}$ \\ ${ }^{a}$ Konkoly Observatory, H-1525 Budapest XII, P.O. Box 67, Hungary \\ ${ }^{\mathrm{b}}$ LAEFF/INTA, P.O. Box 50727, 28080 Madrid, Spain \\ and IAA/CSIC, P.O. Box 3004, 18080 Granada, Spain
}

\begin{abstract}
FG Sagittae is one of the most important key objects for post-AGB stellar evolutionary studies. As a consequence of a final helium shell flash, this unique variable has shown real evolutionary changes on human timescales during this century. The recently observed variations in the emission lines of the surrounding old planetary nebula prove that there is no hot exciting object hidden inside the nebula, thus there is only one central star, namely FG Sge itself. Consequently, we are witnessing the rapid evolution of a single star. Turning to an $\mathrm{R} \mathrm{CrB}$ type variable in the early $90 \mathrm{~s}$, FG Sge also solved the question of the evolutionary state of this type of stars.

Some aspects of the observational history of FG Sge are reviewed in this paper and compared with predictions from model calculation results.
\end{abstract}

Key words: Stars: post-AGB, Stars: evolution, Stars: fundamental parameters, Stars: individual: FG Sge, Stars: oscillations, Stars: variables, Planetary nebulae: individual: He 1-5

PACS: 97.10.Cv, 97.20.Pm, 97.20.Rp, 97.30.-b, 97.30.Hk, 98.38.Ly

\section{Introduction}

FG Sagittae, the central star of the old planetary nebula He1-5 has evolved before our eyes from left to right in the HR diagram, going, in just hundred years, from the hot region of exciting sources of planetary nebulae to the cool red-supergiant domain, thus becoming a newly-born post-AGB star. In 1880

1 E-mail: jurcsik@buda.konkoly.hu

2 E-mail: bmm@laeff.esa.es

Preprint submitted to Elsevier Preprint 18 February 1998 
the spectral type was as early as $\mathrm{O} 3$ and $T_{\text {eff }} \simeq 45,000 \mathrm{~K}$ (van Genderen \& Gautschy 1995). At the beginning of the century the effective temperature was still around $40,000 \mathrm{~K}$, whereas the estimates in the $80 \mathrm{~s}$ and 90 s give $5,000-6,500 \mathrm{~K}$.
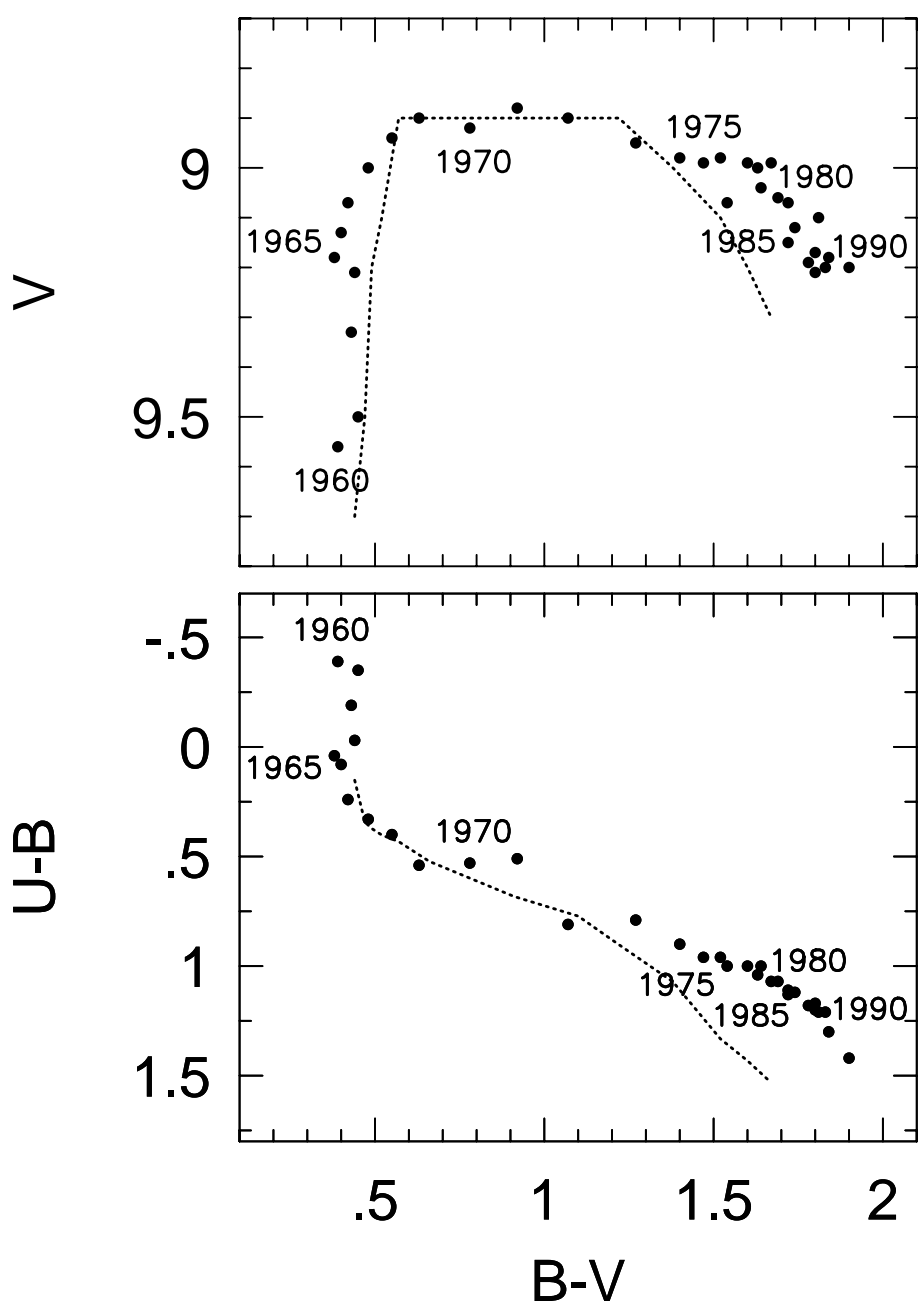

Fig. 1. The evolution of FG Sge in the $\mathrm{V}$ vs. $\mathrm{B}-\mathrm{V}$ and in the $\mathrm{U}-\mathrm{B}$ vs. $\mathrm{B}-\mathrm{V}$ planes. For comparison, the dotted lines show the reddened $\left(\mathrm{E}_{\mathrm{B}-\mathrm{V}}=0.35\right)$ Ia supergiant positions. From the early 70 s the observed surface enrichment of the s-process elements resulted in enhanced line blanketing, which significantly affected the colours. This also led to difficulties in the temperature determinations henceforth.

In order to summarize the rapid changes observed in the physical parameters of the star, Table 1 lists the spectral type, effective temperature and pulsation period since 1930, when the photographic data allowed the first estimate of the period. During the redward evolution the luminosity of the star did not 
change significantly, instead the radius was increasing simultaneously with the temperature variation (see e.g. Table 3 in van Genderen \& Gautschy (1995)). These changes in the size of the extended atmosphere are reflected in the observed increase of the pulsation period. The actual value of the radius depends on the distance assumed, which is, however, uncertain $(2,500-4,000 \mathrm{kpc})$. The determination of $T_{\text {eff }}$, and the spectral type are extremely difficult and depend strongly on the wavelength range or technique used. This has been especially true since the surface chemical composition turned to be peculiar. Because the energy distribution and colours of FG Sge cannot be compared in a straightforward manner with those of standard stars, quite discrepant results were obtained even for the same epoch (e.g. 1985-89 in Table 1).

The evolution of FG Sge can also be followed in the $\mathrm{V}$ vs. B-V and U-B vs. $\mathrm{B}-\mathrm{V}$ planes as shown in Fig. 1. These graphs and the data in Table 1 also show that the speed of the evolution slowed down in the last two decades. A slight blueward evolution was even possible in the late $80 \mathrm{~s}$, but this is difficult to assure due to the uncertainties in the temperature determinations arising from the very complex behaviour of the spectra.

After the pioneering work by Herbig \& Boyarchuk (1968) FG Sge was the subject of many important observational and theoretical studies. Although there is an agreement in that the observed changes are connected with a final He shell flash of a post-AGB object, the details of the phenomenon still conceal many unsolved questions. The comparison of evolutionary calculations for thermally pulsating post-AGB models with the observations yield a mass of $0.61 \pm 0.04 M_{\odot}$ for FG Sge (Blöcker \& Schönberner 1997). To gain a deep insight into the evolutionary history of FG Sge the interested reader should consult e.g. Montesinos et al. (1990) and van Genderen \& Gautschy (1995).

Due to mixing processes accompanying the rapid evolution, the surface chemical composition of FG Sge has also shown drastic changes. In the early $70 \mathrm{~s}$, an enhancement in the abundances of s-process elements was observed (Langer et al. 1974). Later, in the 80s, FG Sge became a carbon star (Iijima \& Strafella 1993). According to the work by Blöcker \& Schönberner (1997) the enrichment of carbon and s-process elements occurred already during the normal AGB phase of FG Sge and cannot be directly connected with processes taking place during the He shell flash observed. In August and September 1992, FG Sge underwent a 4 mag deep dimming (Jurcsik 1992), which was later identified as an R CrB type decline (Jurcsik 1993, Kipper \& Kipper 1993, Gonzalez et al. 1998). Since then, FG Sge has been one of the most active members of this class of variables. Though FG Sge is not as hydrogen deficient as a typical R CrB star is, both its photometric and spectroscopic behaviour support the $\mathrm{R} \mathrm{CrB}$ classification. Witnessing the birth of a new $\mathrm{R} \mathrm{CrB}$ star reveals the evolutionary state of these variables as born-again AGB objects. The onset of the $\mathrm{R}$ CrB type mass loss from the star is, however, in 
Table 1. Summary of the evolution of FG Sge

\begin{tabular}{|c|c|c|c|c|}
\hline Date & Spectral type & $T_{\text {eff }}(\mathrm{K})$ & Ref. & $P$ (days) \\
\hline 1930 & B0 & $\approx 25,000$ & 12 & 5 \\
\hline 1955 & B4 I & $>10,000$ & 1 & 13 \\
\hline 1960 & B9 I & 9,700 & 1 & 20 \\
\hline 1965 & A3 I & 8,700 & 1 & 42 \\
\hline 1966 & $\mathrm{~A} 3-\mathrm{A} 4 \mathrm{I}$ & 8,500 & 1 & \\
\hline 1967 & A5 I & 8,300 & 1 & 52 \\
\hline 1968 & A3 I & 8,$700 ; 9,400$ & 1,2 & \\
\hline 1969 & F0-F2 I & $7,500-7,200 ; 7,700$ & 1,2 & \\
\hline 1970 & $\mathrm{~F} 2-\mathrm{F} 6 \mathrm{I}$ & $7,200-6,500$ & 1 & 70 \\
\hline 1971 & F4-F7 I & 6,$300 ; 7,200$ & 1,2 & 55 \\
\hline 1972 & F6 I & 6,300 & 1 & 53 \\
\hline 1975 & G2 I & 5,$500 ; 6,200$ & 1,2 & 85 \\
\hline 1976 & & 5,900 & 2 & 77 \\
\hline 1977 & G3-G4 I & 5,$300 ; 5,700$ & 1,2 & 85 \\
\hline 1979 & G8-G9 I & 5,000 & 1 & 105 \\
\hline $1980-82$ & G8-K0 I; K0-K2 I & 5,000 & $1,3,5$ & 90 \\
\hline 1983 & G I & 5,600 & 9 & 107 \\
\hline $1985-86$ & F5-G2 I; K0-K2 I & $5,500-6,800 ; 5,500$ & 4,5 & $112-115$ \\
\hline $1986-89$ & F6-F7 I; F8-G2 I; G8-K0 & 6,500 & $6,7,8$ & $100-150$ \\
\hline 1992 & Gp & $5,500-6,000 ; 5,500$ & $10,11,14,15$ & 75 \\
\hline 1994 & & 5,$500 ; 6,500$ & 11,14 & 100: \\
\hline 1995 & & 5,500 & 13 & 74 \\
\hline 1996 & & 5,$400 ; 5,500$ & 14,15 & \\
\hline
\end{tabular}

Spectral type and temperature determinations are from the compilation and results of (1) Acker et al. (1982), and from (2) Stone (1979); (3) Acker (1983); (4) Taranova (1987); (5) Kipper \& Kipper (1989); (6) Montesinos et al. (1990); (7) Arkhipova \& Taranova (1990); (8) Feibelman \& Bruhweiler (1990); (9) Woodward et al. (1993); (10) Stone et al. (1993); (11) Kipper et al. (1995); (12) van Genderen \& Gautschy (1995); (13) Kipper (1996); (14) Gonzalez et al. (1998); (15) this paper.

The pulsation periods prior to 1970 are from van Genderen and Gautschy (1995), those between 1970 and 1994 have been estimated from all the available photometric observations, the value for 1995 is from Guinan (1997, private communication). 
question. This might have occurred even earlier than 1992 as the detection of two circumstellar CO shells by Hinkle et al. (1994) before the first decline indicates.

During the redward evolution, FG Sge became unstable against radial pulsation, thus the pulsational properties also provide useful information on the changes in the surface physical parameters. In a detailed study, van Genderen \& Gautschy (1995) reconstructed the evolutionary and pulsational history of FG Sge in order to deduce its physical parameters. Investigating the changes in the pulsational behaviour coincidental with the occurrence of the decline episodes also help to understand the R CrB phenomenon. The direct observational evidence of chemical evolutionary processes (nucleosynthesis, mixing, dust condensation) and different type of mass loss episodes (planetary nebula, mass ejection connected with the final flash and with the $\mathrm{R}$ CrB behaviour) also give the opportunity to study such phenomena which are closely related with stellar evolution.

In this paper we concentrate in four fundamental aspects which are key issues for understanding the current status of FG Sge and its planetary nebula. In Section 2 the energy distributions before and after the dimming episode are described. Observations with ISOPHOT, the photometer on board ISO (Infrared Space Observatory) are presented for the first time. In Section 3 the problem of the possible presence of a binary system, instead of a single star, in the centre of the nebula is addressed. Section 4 describes the evolution of the pulsation of FG Sge and some features that can be derived from this phenomenon. Section 5 describes FG Sge as an R CrB variable, the evolutionary phase shown by this star since August 1992.

\section{The energy distribution of FG Sge}

The energy distribution of FG Sge has changed according to the spectral type of the central star. The spectroscopic studies of FG Sge started in 1955 (Herbig \& Boyarchuk 1968), at that time the spectral type was close to B4 I. In the last four decades, the spectra have been always similar to those of supergiant stars (see Table 1). The luminosity class ' $\mathrm{I}$ ' that appears in all the classifications is fictitious since the actual structure of the star is different from that of a normal supergiant. Typical masses for $\mathrm{F}$ or $\mathrm{G}$ supergiants lie in the range 10-13 $M_{\odot}$ compared with the estimated mass of FG Sge, namely $0.61 M_{\odot}$. The reason is that the spectral classifications are always done by comparing the spectra of FG Sge with a stellar library and the spectral features of the photosphere of supergiant stars are the closest ones to those shown by FG Sge.

It is very illustrative to compare the energy distributions of FG Sge before and 


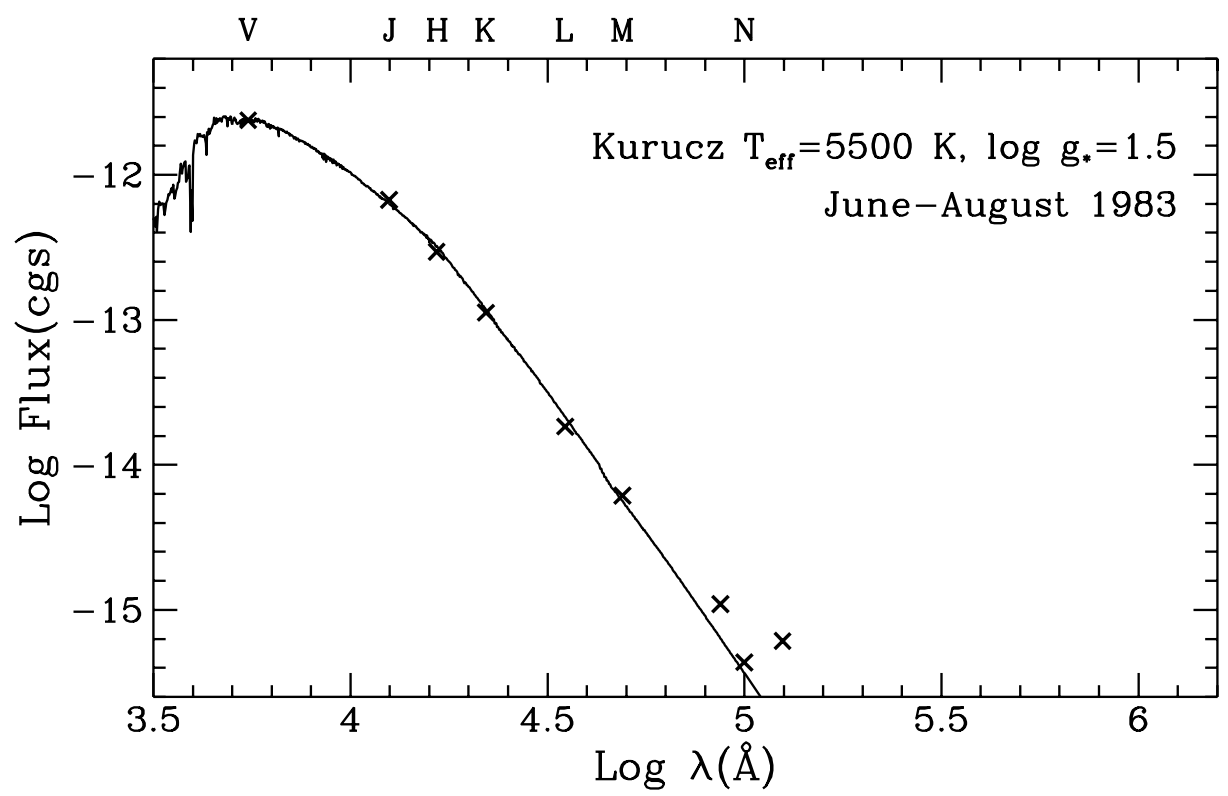

Fig. 2. The energy distribution of FG Sge in 1983.6. Apart from a small discrepancy at longer wavelengths, a purely stellar spectrum fits quite well the observed energy distribution. See text for details.

after the dimming episode that occurred in August-September 1992. All the fluxes shown in Figs. 2 and 3 have been corrected for interstellar absorption using a colour excess $\mathrm{E}(\mathrm{B}-\mathrm{V})=0.40$. In Fig. 2 we can see the photometric VJHKLMN, 7.80 and $12.50 \mu \mathrm{m}$ observations obtained in June-August 1983, transformed into fluxes, and plotted against wavelength. The value adopted for $\mathrm{V}$ was 9.2, the mean magnitude for FG Sge during the decade prior to 1992, the other data have been taken from Woodward et al. (1993). The observed values are represented by crosses and superimposed we have drawn a normalized Kurucz model with $T_{\text {eff }}=5,500 \mathrm{~K}$ and $\log g_{*}=1.5$. The fit, apart from a small discrepancy at longer wavelengths is remarkable. It is clear that the whole energy distribution was completely of stellar origin at that time.

In Fig. 3 we show the energy distribution of FG Sge in two different epochs after the 4-magnitude drop in 1992. In the upper graph the observed fluxes in UBVRI, obtained at Konkoly Observatory, plus observations in HKLMN and $7.80,8.70,9.80,10.30,11.60,12.50$ and $18.00 \mu \mathrm{m}$ (Woodward et al. 1993), both sets in the period 2-11 October 1992, i.e. after the dimming episode, are plotted against wavelength. The energy distribution has changed its shape, showing now a hump corresponding to the dust envelope. A Kurucz model with $T_{\text {eff }}=6,000 \mathrm{~K}$ and $\log g_{*}=1.5$ plus a black body with $T_{\mathrm{BB}}=1,000 \mathrm{~K}$ fits very nicely the whole spectrum. In the lower graph, BVRI photometry taken by Edward Guinan at Villanova University, JHKL' taken during service time at 

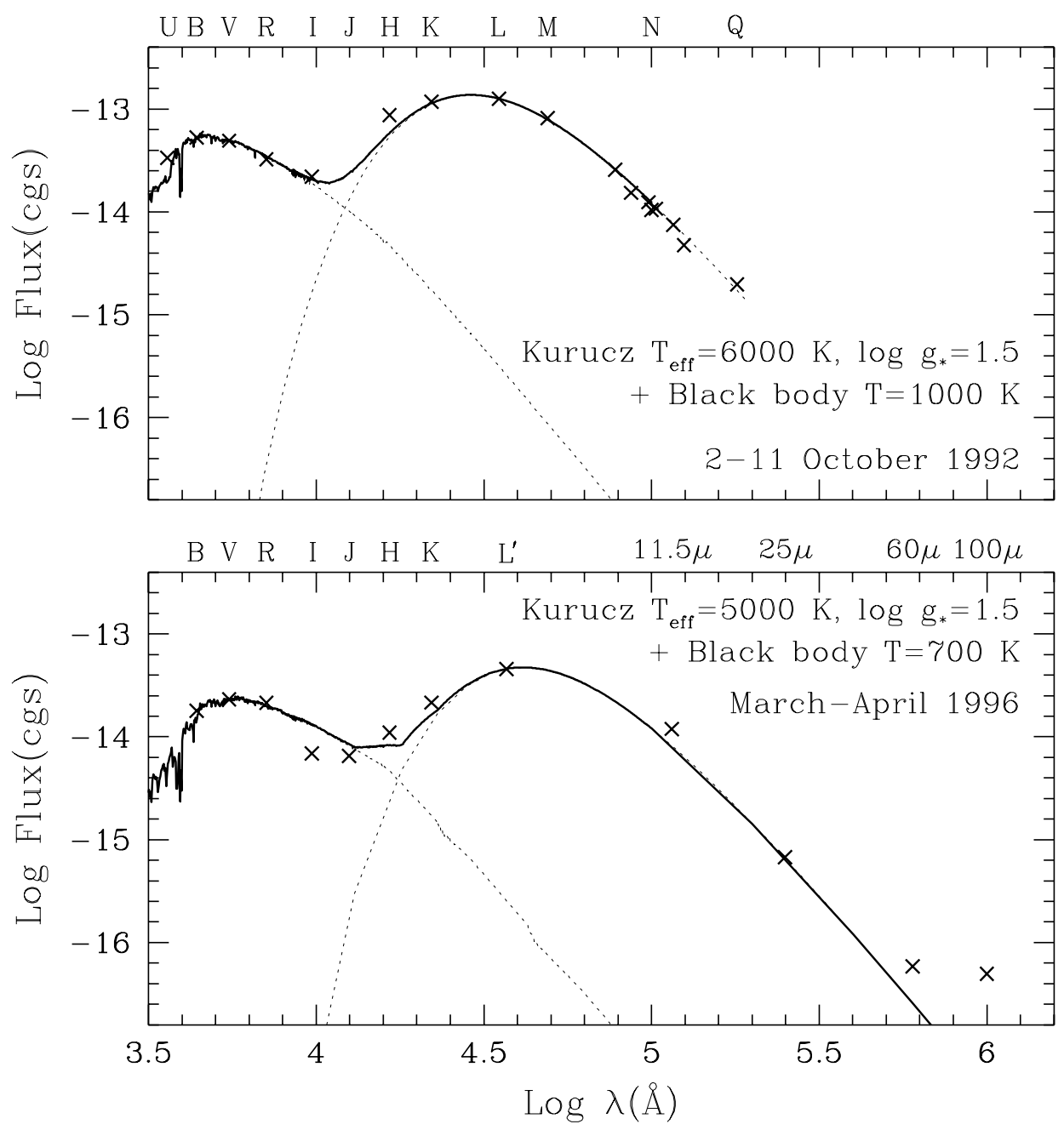

Fig. 3. The energy distribution of FG Sge in 1992.9 (top) and 1996.3 (bottom). The hump at longer wavelengths corresponds to the emission by a dust shell. See text for details.

Observatorio del Teide (Tenerife, Spain) and ISOPHOT observations at 11.5, 25, 60 and $100 \mu \mathrm{m}$, all sets obtained in March-April 1996, are plotted against wavelength. In this case a Kurucz model with $T_{\text {eff }}=5,000 \mathrm{~K}$ and $\log g_{*}=1.5$ plus a black body with $T_{\mathrm{BB}}=700 \mathrm{~K}$ fit nicely the observations.

The results from the fits shown in Fig. 3 have to be taken with caution and only as simple estimates. Neither the wavelength dependence of the internal extinction caused by the dust shell on the stellar light, nor the actual degree of this extinction, are known. An additional problem would be the treatment of the scatter of stellar light by the dust grains. We have assumed in these fits a grey internal extinction law superimposed on the interstellar absorption, and have also neglected any scattering effects. On the other hand, the ISOPHOT calibration is preliminary, so we do not claim that a decrease of $\approx 300 \mathrm{~K}$ has 
occurred in the temperature of the envelope from 1992 to 1996, neither we claim that the central star has also decreased its effective temperature by $1,000 \mathrm{~K}$ between the two dates. The shape and relative intensity of the hump at longer wavelengths affect not only the temperature of the black body to be fitted, but also the stellar spectrum which fits the energy distribution at shorter wavelengths.

We can conclude that the temperature of the central star is around $5,500 \mathrm{~K}$ and the temperature of the condensed dust is around 800-1,000 K. According to Woodward et al. (1993) the corresponding mass in the shell, assuming a dust temperature of $1,000 \mathrm{~K}$ is $M_{\text {dust }} \simeq 3.3 \times 10^{-9} M_{\odot}$.

\section{FG Sge, the central star of the planetary nebula He1-5}

The unique behaviour of FG Sge also raised the possibility that the observed phenomena might be connected with the evolution of not a single but a binary star. The IUE observations which aimed at answering the question of whether FG Sge is really the central star of the surrounding nebula, could not undoubtedly solve the problem. Although a hot companion could not be revealed, it was also clear that the detection limit of the observations did not rule out the possibility of a white dwarf companion (Feibelman \& Bruhweiler 1990). However, if there were a hot ionizing source still present inside the nebula, then no intensity changes of the nebular emission lines would be expected to occur. On the contrary, if FG Sge is the true nucleus of the nebula then this cannot be in an equilibrium state because the effective temperature of the star dropped below $\approx 25,000 \mathrm{~K}$ back in the $20 \mathrm{~s}$. In this case, according to the model calculations of Harrington \& Marionni (1976) and Tylenda (1980), the cooling of the nebula would result in detectable changes of the nebular line intensities in a few decades. But neither the accuracy nor the time span of the earlier measurements made the observation of the expected changes possible.

The drastic dimming of FG Sge in 1992 gave a good opportunity to obtain recent measurements of the nebular line intensities separate from the otherwise too bright stellar light. In 1995 Ingemar Lundström (Lundström 1997) obtained some spectra of the nebula with the Nordic Optical Telescope with a slit position similar to that used by Hawley \& Miller (1978) in the early 70s. In order to compare these new observations with earlier results, Table 2 lists all the available nebular line intensity measurements. It can be clearly seen that there are already obvious changes detected, especially in the [OIII] line intensities. The measured dimming of the $\lambda 4959 \AA$ and $\lambda 5007 \AA$ lines are even more intense than the model calculations would predict. The decline rate of the [OIII] lines, however, strongly depends on the density of the nebula, consequently, slight changes in the initial conditions of the model calculations 
Table 2. Line intensity changes in the planetary nebula around FG Sge

\begin{tabular}{|c|c|c|c|c|}
\hline Date & & 1960 & $1972-1976$ & 1995 \\
\hline Ref. & & (1) & $(2)$ & $(3)$ \\
\hline$\lambda(\AA)$ & line & & $I(\lambda)$ & \\
\hline 3727 & [O II $]$ & 200: & $659-687$ & \\
\hline 3868 & [Ne III $]$ & 30 & $20-42$ & \\
\hline 4101 & $\mathrm{H} \delta$ & & 38 & \\
\hline 4340 & $\mathrm{H} \gamma$ & & $35-56$ & \\
\hline 4363 & [O III] & & $<4$ & \\
\hline 4686 & HeII & $<10$ & $<3$ & \\
\hline 4861 & $\mathrm{H} \beta$ & 100 & 100 & 100 \\
\hline 4959 & [O III] & 100 & $69-71$ & 18 \\
\hline 5007 & [O III] & & $209-269$ & 43 \\
\hline 5755 & {$[\mathrm{~N} \mathrm{II}]$} & & $<3$ & noise level \\
\hline 5876 & HeI & & 13 & 14: \\
\hline 6548 & {$[\mathrm{~N} \mathrm{II}]$} & & $78-96$ & 105 \\
\hline 6563 & $\mathrm{H} \alpha$ & & $284-287$ & 287 \\
\hline 6584 & [N II] & & $294-376$ & 289 \\
\hline 6717 & {$[\mathrm{~S} \mathrm{II}]$} & $30:$ & 42 & 22 \\
\hline 6731 & [S II] & & 34 & 16 \\
\hline
\end{tabular}

References: (1) Herbig \& Boyarchuk (1968); Harrington \& Marionni (1976)

(2) Hawley \& Miller (1978); Kupo \& Leibowitz (1979)

(3) Lundström (1997)

would possible result in satisfactory agreement. Other inconsistencies between the observed line intensity changes and the model predictions (e.g. the S[II] lines have faded away instead of showing temporal brightening as predicted by the models) also indicate some flaws in the theoretical understanding of the processes or in the model parameters applied.

The most important consequence of the detection of intensity changes in the nebular lines is, however, that it clearly shows that FG Sge was the original ionizing source of the nebula, and as such, its present evolution can be undoubtedly connected with that of a single post-AGB star. 


\section{FG Sge, the pulsating star}

Besides the long-term photometric and spectroscopic changes, FG Sge also exhibits brightness fluctuations on shorter timescales due to pulsation. Not only the temperature and the radial velocity were shown to be varying during the pulsational cycles (Jurcsik \& Szabados 1981) but also the depth of the C2 Swan bands which were first detected on the spectrum in 1981 (Iijima 1996). As the pulsational properties also reflect the structure and the temporal physical parameters of the atmosphere, studying the pulsational behaviour might give an independent means to determine both the direction and the speed of the evolution. However, the semiregular nature of the pulsation makes such conclusions somehow uncertain.

In the top panel of Fig. 4 all the photoelectric V observations of FG Sge between 1969 and 1992 are plotted. Thanks mostly to the continuous monitoring of Arkhipova and her collaborators (Arkhipova 1994, and references therein), besides the seasonal gaps, the pulsation was well followed during this period. The middle panel shows the mean cycle length observed in each year. Although as a result of the irregularities in the light curve, the annual periods can be only determined with an accuracy of some days, still some kind of cyclic behaviour (with a timescale of about $4-5$ years) in both the amplitude and the period increase is clearly seen. Due to the large extent of the diluted atmosphere and also to the mixing processes accompanying the rapid evolution which led to drastic changes in the surface chemical composition during the past decades (Langer et al. 1974, Kipper et al. 1995, Gonzalez et al. 1998), there are large uncertainties in estimating even the relative changes of both the temperature and the luminosity of the star. Therefore, the fluctuations in the period increase might simply reflect that the redward evolution of the star was not strictly steady. The observed fluctuations in the amplitude, however, also raised the possibility that different pulsational modes were simultaneously excited. R CrB, and RY Sgr, the photometrically best studied R CrB stars, both have two or more periods 30-60 day long, with stable or varying amplitudes. Linear non-adiabatic radial pulsation analyses of model sequences corresponding to the observed evolution of FG Sge (van Genderen \& Gautschy 1995) show that besides the fundamental, many of the lower overtones are also unstable in the temperature domain $(5,000-8,000 \mathrm{~K})$ of interest.

The redward evolution of the star was, however, so rapid that the timescale of the changes in the physical parameters of the atmosphere was hardly longer than that of the pulsation itself. As a result, period changes with some days/year rate were expected to occur. This, and also the possible variations in the amplitudes, make it difficult to reveal different modes. Whereas Jurcsik \& Szabados (1989) were able to describe the main characteristics of some segments of the light curve assuming two periods with ratio of about 0.9 , they 
failed to fit all the features of the light curve. An alternative explanation of the light-curve characteristics is that the nonlinearities of the highly non-adiabatic extended atmosphere lead to chaotic behaviour of the pulsation.

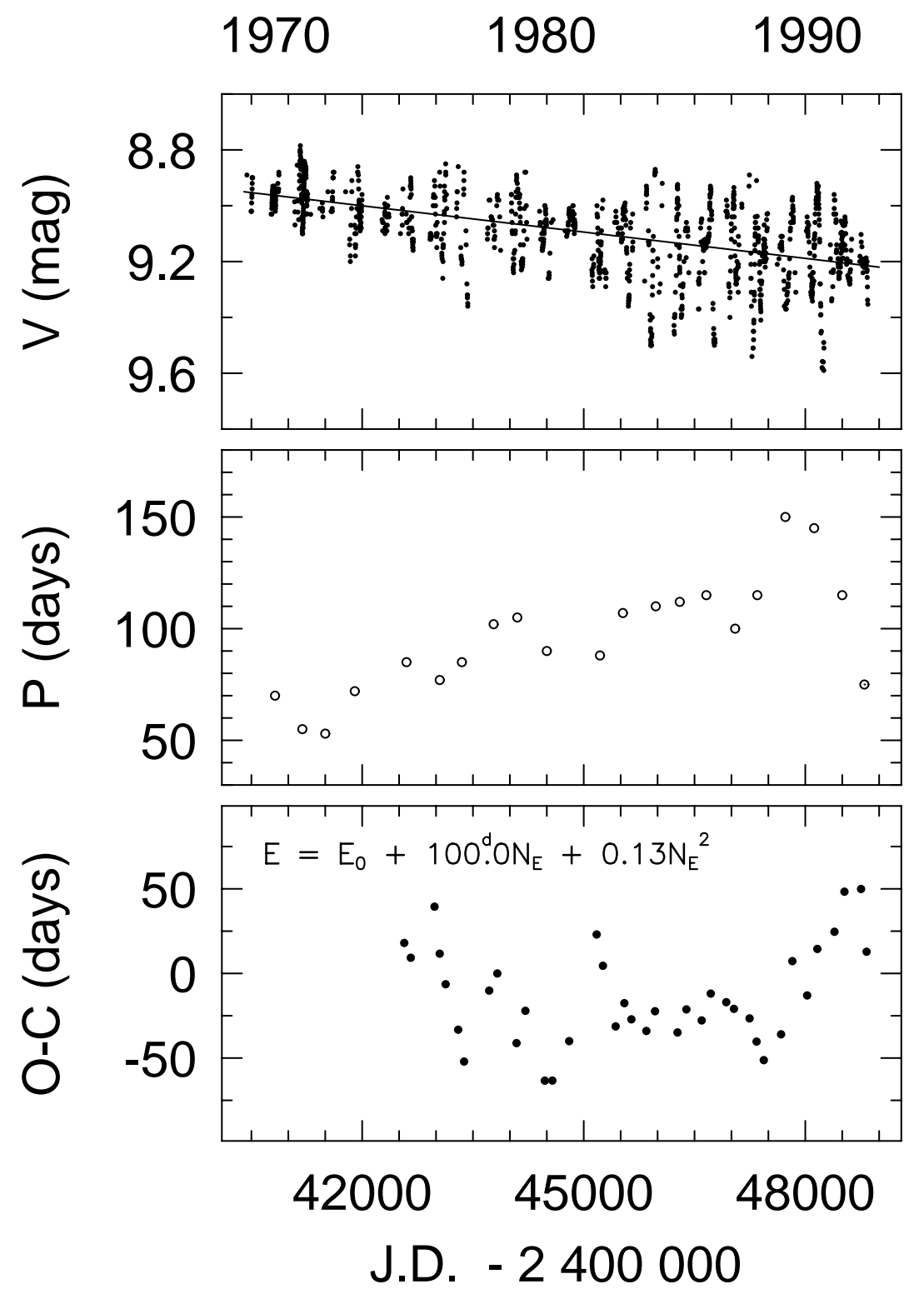

Fig. 4. V light curve (top), yearly average pulsation period of FG Sge from 1969 till the onset of the 1992 deep decline (middle) and a trial $\mathrm{O}-\mathrm{C}$ diagram assuming a linear increase in the period (bottom), all plotted against time.

Though it is not known when the R CrB type activity of FG Sge have actually started preceding the first decline observed, it is also possible that the drop in both the period and the amplitude of the pulsation in 1991-92 (see Fig. 5) is related to the onset of the $\mathrm{R}$ CrB type behaviour. The bottom panel in Fig. 4 shows the $\mathrm{O}-\mathrm{C}$ values of the light maxima calculated by using an 
ephemeris of a linearly increasing 100-day period. It can be seen that the $\mathrm{O}-\mathrm{C}$ values are commensurable with the period over the whole interval plotted. This result remains unchanged if any other trial period with linear or cubic changes are used. Therefore, we cannot truly decide whether the changes in the light curve properties that occurred preceding the 1992 decline were indeed more significant than any of the other fluctuations observed earlier.

The pulsation model results give rise to even two possible explanations for a period drop in the temperature range valid for FG Sge at that time. (There are, however, some doubts about the adequacy of linear pulsation models which, moreover, were calculated assuming higher mass values than Blöcker \& Schönberner (1997) derived for FG Sge.) All the different mass and luminosity model sequences calculated by van Genderen \& Gautschy (1995) show a bump with a sharp decline at around $6,300 \mathrm{~K}$ of the fundamental mode periods. Thus the drop in the period might be a mere consequence of further slight cooling of the star. At even lower temperatures, another significant period increase occurs which might indicate that the observed period decrease could be related to a switching from the fundamental mode to the also unstable first overtone. Unfortunately, because of the uncertainties in both the pulsation model results and the temperature determinations, it is not possible at this moment to draw straightforward conclusions from the comparison of the models with the observations.

\section{FG Sge, the $\mathrm{R}$ CrB variable}

Although some indirect evidence of connection between planetary nebulae and R CrB stars were already found (faint PN-like halos around some R CrB stars, the $\mathrm{H}$ deficient knots in A30 and A78), the evolutionary state of R CrB stars has not been undoubtedly cleared yet (see Clayton (1996) for details on $\mathrm{R}$ CrB type stars). The recent photometric behaviour and the spectroscopic characteristics of FG Sge are very similar to those of an R CrB star (see also Gonzalez et al. 1998). Only the insufficient $\mathrm{H}$ deficiency in the atmosphere poses some doubt about the present classification as an $\mathrm{R} C \mathrm{CB}$ variable but there are other $\mathrm{R}$ CrB stars which are not significantly $\mathrm{H}$ deficient, too. It was shown that the decline activity of the $\mathrm{R}$ CrB stars correlates with the $\mathrm{H}$ content of the atmosphere in the sense that the less $\mathrm{H}$ deficient objects are the most active ones (Jurcsik 1996). According to this empirical relationship, FG Sge was expected to show very frequent decline episodes, and indeed, following the first 4-mag deep minimum in the autumn of 1992, no full recovery of the star ensued; instead, consecutive minima of different depths occurred. The photometric history of the last years was shown in Fig. 1 of Gonzalez et al. (1998) using visual photometric measurements of amateur astronomical associations. 


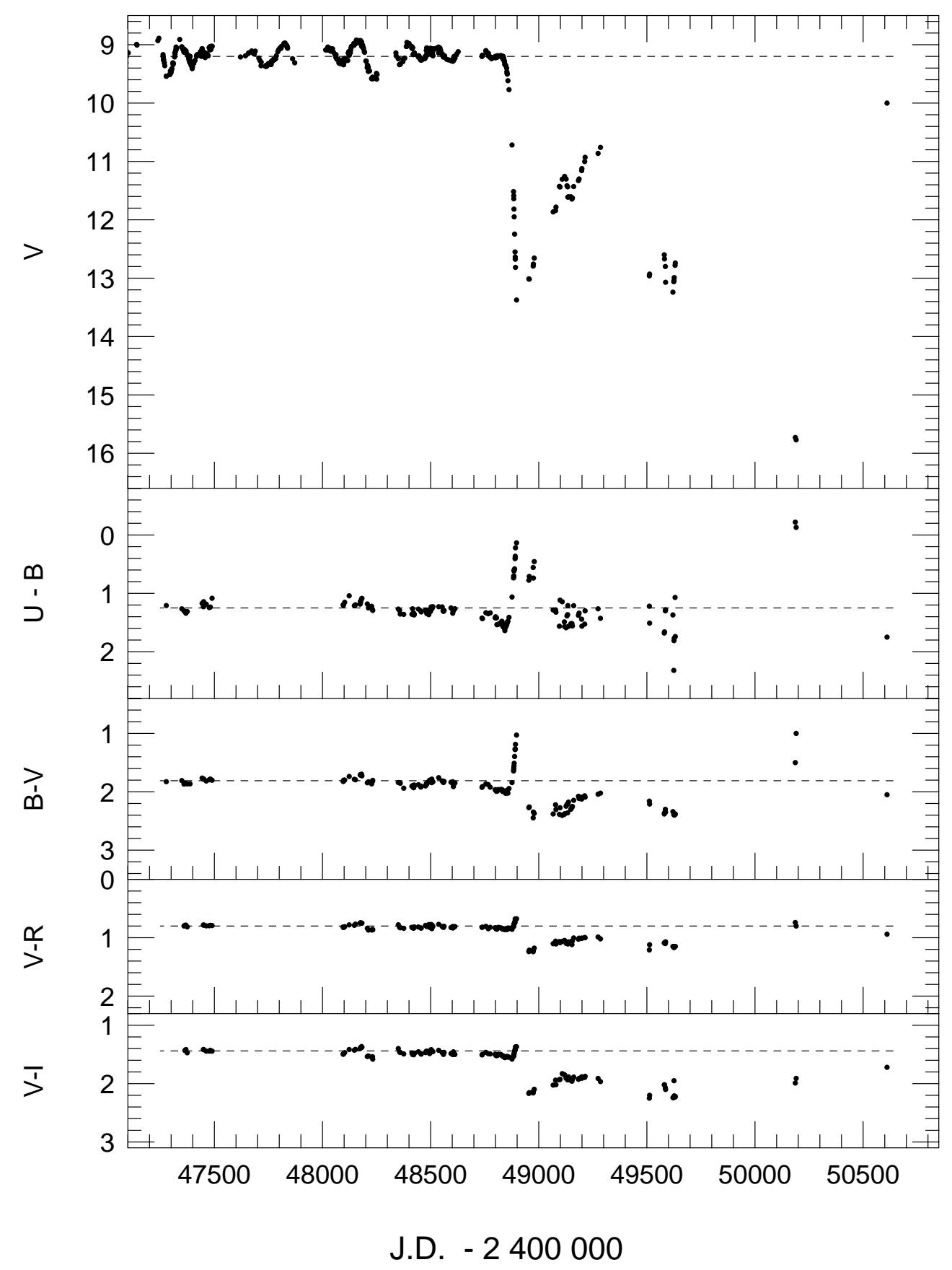

Fig. 5. Photometric observations of FG Sge plotted against time. Preceding the first R CrB type decline in 1992 the photoelectric V observations of Arkhipova (1993, 1994), van Genderen (1994) and Jurcsik (1992) are shown. From the onset of the first decline and for all the colour indices only the photoelectric and CCD observations obtained at Konkoly Observatory are plotted. 


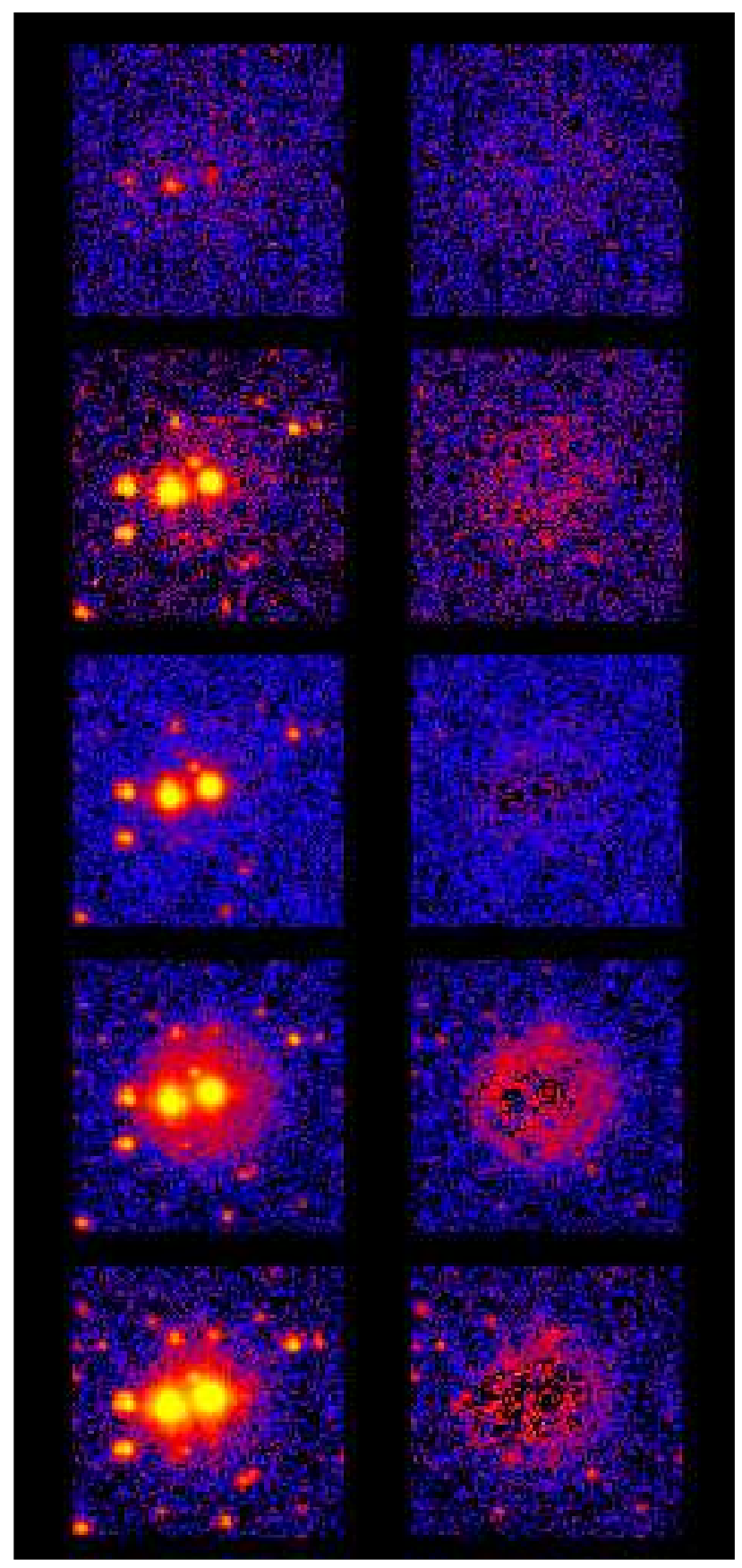

Fig. 6. The five frames in the left hand side show, from top to bottom, UBVRI CCD observations of FG Sge on 30 September, 1994. FG Sge, the rightermost object of the two brighter stars, was 17.2, 15.4, 13.0, 11.8 and 10.8 mag faint in the U,B,V,R and I bands, respectively. The pictures in the right hand side show the same frames after the point spread function removal of the brighter stars. In these images the fossil emission of the surrounding planetary nebula can be seen. 
Most of the R CrB stars are also pulsating variables and in some cases (RY Sgr, V854 Cen) the onset of the deep minima seems to be linked to a specific phase of the pulsation. This would consequently mean that the condensation of the dust particles in the ejected material which is responsible for the observed declines, has to take place in the vicinity of the atmosphere. Such findings thus have major influence on the interpretation of the $\mathrm{R} \mathrm{CrB}$ phenomenon. Because the pulsation of FG Sge can be followed easily as the amplitude is large enough, and also because it seems that the decline episodes occur quite frequently, FG Sge can be a very important key object in settling the question of the link between pulsation and decline. Arkhipova (1996) found an ephemeris $(\mathrm{P} \approx 115$ day with a slight period decrease) which predicted minima of the pulsation coinciding with the four deep minima observed between September 1992 and December 1995. This ephemeris, however, failed to show such a coincidence for the subsequent deep minimum in 1996. Moreover, although three cycles of the pulsation with periods around 110 days were certainly observed during the recovery phase from the 1992 minimum, afterwards both the timescale and the amplitude of the pulsation seemed to change significantly again. Accordingly, there are some doubts that evidences of a causal link between pulsation and declines have been actually observed. We also mention that the very frequent declines in V854 Cen make the detection of its pulsation difficult, so in that case there is also some uncertainty about the connected phases of the two types of light variations.

Another interesting feature of the R CrB-like behaviour of FG Sge is that, according to the multicolour observations of its early decline phases, most, if not all, of these were 'blue' type declines (see Fig. 5, and also Arkhipova 1996 and Guinan 1998). Although the photometric colours during the decline phase are somewhat uncertain when determined from observations using large apertures, the small aperture size (5"-10") used at Konkoly Observatory and also the advantages of the CCD observations and reduction techniques, make the blueing of the colours during the early phases of the 1992 and 1995 declines unquestionable. The CCD observations in 1994 were obtained during recovery phases and during a mid-decline phase to a smaller secondary minimum. To see the problems of obtaining accurate photometric measurements, in Fig. 6 one of the CCD UBVRI observations is shown. For each colour the same frames are also shown in the right after the removal of the point spread functions of the brighter stars. The planetary nebula surrounding FG Sge became clearly visible in these pictures especially in the B,R and I bands. FG Sge had similar brightness ( $\mathrm{V}=13.0 \mathrm{mag}$ ) and colours at the time of this observation as its optical companion 8" to the left. The observed changes of the colours during the decline phase in $\mathrm{R}$ CrB stars are mainly caused by the obscuration and also the scatter of the dust particles but intense changes in chromospheric emissions should also have important effect especially in the early decline phase. The statistics of the colours of the declines (see e.g. Cottrell 1998) shows that both red and blue type declines of the same object are observed, therefore if the 
future declines of FG Sge keep on being blue type ones, this would mean a special uniqueness in the $\mathrm{R}$ CrB character of FG Sge.

\section{Final remarks}

There are no doubts that FG Sge is one of the most fascinating stellar objects (if not the most fascinating one!) ever observed. Its history is full of surprises, and has provided valuable clues and contributions to our present knowledge of the late stages of stellar evolution. Although the rough scheme of the further evolution that FG Sge would have to follow is thought to be known, we think that no one should dare to forecast what the next surprise FG Sge will have in store for those observers who persistently keep an eye on this remarkable object.

\section{Acknowledgements}

We are indebted to Ingemar Lundström for his contribution in obtaining the spectra of the nebula at our request, and providing the recent line intensities and also to Ed Guinan for part of the photometric data presented in Section 2. The IAU and LOC grants that made it possible for us to attend the XXIIIrd IAU General Assembly are acknowledged. J.J. would like to thank the support from the Hungarian OTKA grants T-024022 and T-019640. She is also grateful to László Szabados for his continuous interest and help. B.M. would like to acknowledge the Spanish DGICYT which funded partially this work through grant PB94-1275. Part of this work is based on service time observations made with the Carlos Sánchez telescope of the Observatorio del Teide (Tenerife), operated by the Instituto de Astrofísica de Canarias.

\section{References}

Acker, A., 1983, A\&AS 54, 293

Acker, A., Jaschek, M., \& Gleizes, F., 1982, A\&AS 48, 363

Arkhipova, V.P., 1993, Pis'ma Astron. Zh. 19, 593

Arkhipova, V.P., 1994, Pis'ma Astron. Zh. 20, 919

Arkhipova, V.P., 1996, Pis'ma Astron. Zh. 22, 828

Arkhipova, V.P., \& Taranova, O.G., 1990, Pis'ma Astron. Zh. 16, 808 
Blöcker, T., \& Schönberner, D., 1997, A\&A 324, 991

Clayton, G.C., 1996, PASP 108, 225

Cottrell, P., 1998, these proceedings

Feibelman, W.A., \& Bruhweiler, F.C., 1990, AJ 100, 1248

van Genderen, A.M., 1994, A\&A 284, 465

van Genderen, A.M., \& Gautschy, A., 1995, A\&A 294, 453

Gonzalez, G., Lambert, D.L., Wallerstein, G., Rao, K.N, Smith, V., \& McCarthy, J.K, 1998, ApJ Suppl. 114, 133

Guinan, E.F., 1998, these proceedings

Harrington, J.P., \& Marionni, P.A., 1976, ApJ 206, 458

Hawley, S.A., \& Miller, J.S., 1978, ApJ 221, 851

Herbig, G., \& Boyarchuk, A., 1968, ApJ 153, 397

Hinkle, K.H., Joyce, R.R., \& Smith, V., 1994, AJ 109, 808

Iijima, T., 1996, MNRAS 283, 141

Iijima, T., \& Strafella, F., 1993, IBVS No. 3959

Jurcsik, J., 1992, IBVS No. 3775

Jurcsik, J., 1993, Acta Astronomica 43, 353

Jurcsik, J., 1996, Acta Astronomica 46, 325

Jurcsik, J., \& Szabados, L., 1981, Acta Astronomica 31, 213

Jurcsik, J., \& Szabados, L., 1989, Astrophys. \& Space Sci. 153, 45

Kipper, T., 1996, IBVS No. 4346

Kipper, T., \& Kipper, M., 1989 in Evolution of Peculiar Red Giants Eds.: H.R. Johnson and B. Zuckerman, IAU Coll 106, p.146

Kipper, T., \& Kipper, M., 1993, A\&A 276, 389

Kipper, T., Kipper, M., \& Klochkova, V.G., 1995, A\&A 297, L33

Kupo, I., \& Leibowitz, E.M., 1979, A\&A 71, 102

Langer, G., Kraft, R.P., \& Anderson, K.S., 1974, ApJ 189, 509

Lundström, I., 1997, private communication

Montesinos, B., Cassatella, A., González-Riestra, R., Fernández-Castro, T., Eiroa, C., \& Jiménez-Fuensalida, J., 1990, ApJ 363, 245

Stone, R.P.S., 1979, PASP 91, 389 
Stone, R.P.S., Kraft, R.P., \& Prosser, C.F., 1993, PASP 105, 755

Taranova, O.G., 1987, Pis'ma Astron. Zh. 13, 891

Tylenda, R., 1980, Acta Astronomica 30, 433

Woodward, C.E., Lawrence, G.F., Gehrz, R.D., Jones, T.J., Kobulnicky, H.A., Cole, J., Hodge, T., \& Thronson, H.A. Jr, 1993, ApJ 408, L37 